\title{
ANIMAL RESEARCH PAPER In situ and in vitro estimation of mineral release from common feedstuffs fed to cattle
}

\author{
D. ZANETTI ${ }^{1 *}$, A. C. B. MENEZES ${ }^{1}$, F. A. S. SILVA ${ }^{1}$, L. F. COSTA E SILVA ${ }^{1}$, P. P. ROTTA ${ }^{1}$, \\ E. DETMANN ${ }^{1}$, T. E. ENGLE ${ }^{2}$ AND S. C. VALADARES FILHO ${ }^{1}$ \\ ${ }^{1}$ Department of Animal Science, Universidade Federal de Viçosa, PH Rolfs St, 36570-900, Minas Gerais, Brazil \\ ${ }^{2}$ Department of Animal Science, Colorado State University, 350 W Pitkin St, 80523, Colorado, USA
}

(Received 23 March 2017; revised 3 May 2017; accepted 19 May 2017;

first published online 13 June 2017)

\section{SUMMARY}

The objective of the current study was to quantify the dry matter (DM) digestibility, and total ash (TA) and mineral release from 12 concentrate and 12 forage feedstuffs commonly fed to cattle using in situ and in vitro methods. Concentrate and forage feedstuffs were incubated in the rumen of ruminally cannulated beef bulls at eight different time points. Two different trials were conducted for concentrates and forages, with maximum incubation time of 72 and $120 \mathrm{~h}$, respectively. The residue from samples incubated for $24 \mathrm{~h}$ were treated with pepsin and hydrochloric acid to simulate abomasum digestion in vitro. The initial and residual samples after in situ and in vitro incubations were measured. An asymptotic model was adopted for estimating solubility of minerals, disappearance rate of DM, and TA. Correlations between feedstuff contents and mineral release were evaluated. Residual samples from rumen fermentation after $24 \mathrm{~h}$ were incubated in simulated abomasal conditions and mineral release was measured. Cluster analysis was performed to group feedstuffs in relation to TA release. Large variability was observed between concentrate and forage feedstuffs for all constituents analysed. Large variability was observed for the effective ruminal degradation of TA and individual mineral release. When feedstuffs were clustered according to the immediately soluble fraction ('a'), the insoluble by potentially releasable fraction (' $b$ ') and the release rate of ' $b$ ' (' $\left.k d^{\prime}, / h\right)$ estimates of TA ruminal release, four groups were identified. From group ' 1 ' to group ' 4 ', an increase in the soluble fraction and a reduction in both moderate releasable fraction and release rate was observed. Neutral detergent fibre content had a negative correlation with mineral release in the rumen, while mineral content had a positive correlation. These results demonstrate that mineral solubilization in the digestive tract is not the limiting factor for mineral absorption from the feedstuffs tested.

\section{INTRODUCTION}

The majority of nutrient recommendation publications and ration balancing programmes for cattle (USA: dairy cattle, NRC 2001; beef cattle, NRC 2016; United Kingdom: ARC 1980; AFRC 1993; and Brazil: BR-Corte, Valadares Filho et al. 2010) do not consider the inherent mineral contents from feedstuffs, or do not give a recommendation of the absorption coefficient for trace minerals within feedstuffs. Therefore, minerals are typically supplemented to beef cattle diets without taking into consideration

* To whom all correspondence should be addressed. Email: diego. zanetti@ufv.br, diegozanetti_@hotmail.com the inherent minerals within the feedstuffs (Spears 1996). Despite this, two points may be considered in this context for reducing mineral input for cattle. First, in most tropical areas, cattle depend exclusively on forages to meet their mineral requirements (Perdomo et al. 1977; Ibrahim et al. 1998; Costa e Silva et al. 2015; Costa et al. 2016). Second, when mineral supply in a production system is reduced, environmental excretion and costs are reduced (Khorasani et al. 1997; Humer \& Zebeli 2015).

Some researchers (Esser et al. 2009; Prados et al. 2016; Zanetti et al. 2017) have demonstrated that animals fed typical feedlot diets can meet their mineral requirements exclusively from basal feed 
ingredients. This is made possible because the mineral content in feedstuffs is, in general, high (Playne et al. 1978). Mineral bioavailability in feedstuffs is affected by the mineral distribution in plant cells, the form of the mineral, association with cell components, and interaction with other minerals (Čerešnáková et al. 2005; Pogge et al. 2014; Berrett et al. 2015). Mineral availability in the rumen depends on mineral content, fibre content and passage rate (Flachowsky \& Grün 1992; Berrett et al. 2015). Furthermore, the solubility of minerals in general is increased under low $\mathrm{pH}$ conditions. Therefore, mineral content in feedstuffs can be divided into three fractions: very soluble, moderately soluble (minerals that are predominately associated with the fibre and/or protein fractions of the feedstuff), and un-releasable or insoluble minerals (Spears 1994).

Minerals that are not released from feedstuffs are unavailable for absorption. Minerals released from feedstuffs incubated in rumen have been reported to be highly correlated with mineral availability to the animal (Flachowsky et al. 1994). Therefore, this technique is an inexpensive, simple, rapid and reproducible technique that may allow absorption coefficients for minerals contained in feedstuffs to be estimated (Olubobokun et al. 1990). Therefore, the current study hypothesized that a proportion of mineral content within feedstuffs is released under ruminal and abomasal digestive conditions and these values should be considered in formulating diets for ruminants. The objectives were to evaluate the extent of mineral release under ruminal and simulated abomasal conditions.

\section{MATERIAL AND METHODS}

Two experiments were conducted in the Experimental Feedlot of the Animal Science Department of the Universidade Federal de Viçosa, Viçosa, MG, Brazil, following the recommendations of the Ethics Committee for Animal Use and Care. Twenty-four samples of feedstuffs were evaluated (12 concentrate and 12 forage typically fed to beef cattle) to determine mineral release.

\section{Concentrate trial}

Concentrate feedstuffs were divided into two groups where six ingredients were considered as energy concentrates (i.e. low protein content): ground maize (Zea mays L.), ground ear maize (Zea mays L.), ground sorghum (Sorghum vulgare), rice bran (Oryza sativa), soybean hulls (Glycine max (L.) Merr) and wheat bran (Triticum aestivum) while the other six ingredients were considered as protein concentrates (i.e. high protein content): maize gluten (Zea mays L.), cottonseed meal (Gossypium hirsutum), ground bean (Phaseolus vulgaris L.), peanut meal (Arachis hypogaea L.), soybean meal (Glycine max (L.) Merr) and sunflower meal (Helianthus annuus).

Concentrates were randomly divided into four groups and feedstuffs were incubated in the rumen of four cannulated crossbred bulls. The procedure of incubation was conducted four times, and within each of these periods, each concentrate group was incubated in the rumen of a different bull as part of a Latin square design. The bulls received ad libitum access to a diet composed of: $500 \mathrm{~g}$ maize silage $/ \mathrm{kg}$ dry matter (DM) and a concentrate supplement composed of $800 \mathrm{~g}$ ground maize $/ \mathrm{kg}$, $86 \mathrm{~g}$ soybean meal $/ \mathrm{kg}$, $60 \mathrm{~g}$ wheat bran $/ \mathrm{kg}$, $14 \mathrm{~g}$ urea $/ \mathrm{kg}, 1.0 \mathrm{~g}$ ammonium sulphate/kg, $9.0 \mathrm{~g}$ salt $/ \mathrm{kg}, 9.0 \mathrm{~g}$ mineral premix $/ \mathrm{kg}, 15 \mathrm{~g}$ sodium bicarbonate/kg and $5 \mathrm{~g}$ magnesium oxide/ $\mathrm{kg}$. The diet contained $120 \mathrm{~g}$ crude protein $(\mathrm{CP}) / \mathrm{kg}$ DM.

\section{Forage trial}

The forages examined in the current experiment were maize silage (Zea mays L), brachiaria decumbens grass (Urochloa decumbens), elephant grass (Pennisetum purpureum), Brachiaria humidicola grass (Urochloa humidicola), Marandu grass (Urochloa brizantha Cv Marandu), brachiaria MG-4 grass (Urochloa brizantha cv MG-4), Mombaça grass (Panicum maximum cv Mombaça), Mulato grass (hybrid Urochloa decumbens $\times$ Urochloa ruziziensis), peanut forage (Arachis pintoi), Tanzania grass (Panicum maximum cv Tanzânia), Tifton 85 hay (Cynodon spp.) and Brachiaria Xaraes grass (Urochloa brizantha cv Xaraés).

Samples of feedstuffs, except maize silage, were collected $45 \mathrm{~d}$ after regrowth, at the Agrostology Sector from Animal Sciences Department at Universidade Federal de Viçosa, Viçosa, MG, Brazil. The $45 \mathrm{~d}$ of regrowth, during the transition between rainy and dry seasons, was not irrigated, as usual for Brazilian pastures. The samples (leaves and stems) were harvested $10 \mathrm{~cm}$ above the soil. All samples were oven-dried at $55^{\circ} \mathrm{C}$ for $72 \mathrm{~h}$. Forages were randomly divided into three groups and incubated in the rumen of three cannulated crossbred bulls. 
The procedure of incubation was replicated three times and within each of these periods, each forage type was incubated in a different bull as part of a Latin square design. The bulls had ad libitum access to a diet that contained $1000 \mathrm{~g} / \mathrm{kg}$ elephant grass containing $161 \mathrm{~g} \mathrm{CP/kg} \mathrm{DM.}$

\section{Ruminal incubation}

All samples were ground through a 2-mm sieve using a knife mill (TECNAL, Piracicaba, São Paulo, Brazil) for in situ ruminal incubation. Nylon bags (Sefar Nitex, Switzerland; $50-\mu \mathrm{m}$ porosity, $400 \mathrm{~cm}^{2}$ surface area) were individually identified. Six grams of each feedstuff were weighed into each bag and incubated in each animal. The bag surface area to mass ratio was $15 \mathrm{mg} / \mathrm{cm}^{2}$. The number of bags varied as a function of incubation time to guarantee enough residual samples after incubation (i.e. more bags per sample were incubated for the longer incubation times relative to shorter incubations times). The incubation times were $0,2,4,8,16,24,48$ and $72 \mathrm{~h}$ for concentrates and $0,3,6,12,24,36,48,72$ and $120 \mathrm{~h}$ for forages. The times were different for concentrate and forage feedstuffs due different degradation rates. Samples were incubated in the rumen by attaching the bags to a steel chain with a weight at the end to allow for continual immersion within the ruminal contents.

Bags were placed into the rumen in reverse order of incubation hours so that all bags were removed at the same time for washing. After the incubation period, bags were washed by hand with running cold tap water and the end-point for washing was when the rinsing water had a high clarity. The bags for time 0 were not incubated in the rumen, but as with incubated bags they were rinsed in running water. Samples were oven-dried at $55^{\circ} \mathrm{C}$ for $72 \mathrm{~h}$. After drying, bags were placed in an oven at $105^{\circ} \mathrm{C}$ for $2 \mathrm{~h}$ and weighed. Residual samples in bags were used to estimate the parameters of ruminal DM degradation, and total ash (TA) and mineral release. Residues of each feedstuff were removed from nylon bags and placed in a labelled plastic bag, to obtain a sample of each feedstuff per animal/incubation time.

\section{Abomasal digestion simulation}

Residual samples of ruminal incubation at $24 \mathrm{~h}$ were submitted to a simulation of abomasal digestion as described by Berrett et al. (2015). In total $400 \mathrm{mg}$ of sample was weighed into plastic tubes and $15 \mathrm{ml}$ of a solution of $50 \mathrm{~g} / \mathrm{l}$ pepsin and $1.2 \mathrm{~N} \mathrm{HCl}$ (hydrochloric acid) was added to each tube to simulate abomasal digestion conditions. Tubes were then allowed to incubate in a water bath at $39{ }^{\circ} \mathrm{C}$ with gentle swirling every $15 \mathrm{~min}$. After $1 \mathrm{~h}$, residual content was filtered through ashless filter paper (CAT no. 1541-090, Whatman, General Electric). The residual content was oven-dried at $105^{\circ} \mathrm{C}$ for $12 \mathrm{~h}$, and analysed for ash and mineral content.

\section{Analytical procedures}

All feedstuffs were analysed for DM (method 934.01), TA (method 930.05), and total nitrogen $(\mathrm{N}$, method 981-10), according to the AOAC protocols (AOAC 2012). Crude protein content was obtained by multiplying total nitrogen content by $6 \cdot 25$. Neutral detergent fibre (NDF) was obtained according to Mertens (2002) without addition of sodium sulphite and with addition of a thermostable $\alpha$-amylase. Neutral detergent fibre was expressed in ash- and proteinfree basis.

Mineral analysis was performed on all samples (initial and residual) by weighing the samples into pre-weighed acid-washed crucibles then drying the samples in a forced-air oven at $105^{\circ} \mathrm{C}$ for $12 \mathrm{~h}$. After the drying period, samples were reweighed to measure DM content. Samples were then placed in muffle furnace and ashed at $600{ }^{\circ} \mathrm{C}$ for $12 \mathrm{~h}$. Samples were removed and placed in a desiccator for $30 \mathrm{~min}$ to cool. Samples were then weighed and re-suspended with $5 \mathrm{ml}$ of $\mathrm{HCl}(1 \cdot 2 \mathrm{~N} \mathrm{HCl})$. During re-suspension the crucibles were heated using a heat plate. Mineral concentrations (calcium [Ca], phosphorus $[\mathrm{P}$,$] sodium [\mathrm{Na}]$, potassium $[\mathrm{K}]$, magnesium $[\mathrm{Mg}]$, sulphur $[\mathrm{S}]$, copper $[\mathrm{Cu}]$, zinc $[\mathrm{Zn}]$, manganese $[\mathrm{Mn}]$, cobalt $[\mathrm{Co}]$ and iron [Fe]) of the samples were analysed through inductively coupled plasma optical emission spectroscopy methods (Optima 7300 DV, Perkin Elmer; Braselton et al. 1997).

\section{Statistical analysis}

A Latin square experimental design was used to assist and organize the information collected in the field, allowing for measurement of degradation of different feedstuffs without the confounding effect of animal. The objective was to control sources of variation and to avoid bias without estimating variability. The Latin square design, in this case, was not used as a 
way to analyse data for comparative differences among feedstuffs, animals, or collection periods, but to collect source data to generate the following equations.

The profiles for DM degradation, TA and mineral release in the rumen were estimated using the asymptotic model of Ørskov \& McDonald (1979). The following model was used to estimate the parameters of degradability:

$$
Y=a+b \times\left(1-e^{(-k d \times t)}\right)
$$

where $Y$ is fraction degraded or released in the time $t(\mathrm{~g} / 100 \mathrm{~g})$, of DM, TA or individual mineral; ' $a$ ' is the immediately soluble fraction $(\mathrm{g} / 100 \mathrm{~g})$; ' $b$ ' is the insoluble but potentially degradable/releasable fraction $(\mathrm{g} / 100 \mathrm{~g})$; ' $\mathrm{e}$ ' is the Euler's number $(e=$ $2 \cdot 71828183 \ldots)$ and $k d$ is degradation/releasing rate of ' $b$ ' (/h). The effective degradation/release was estimated based on the model of Ørskov \& McDonald (1979):

$$
E=a+\frac{b \times k d}{k d+k p}
$$

where $E$ is effective degradation (or released) fraction, of DM, TA or each mineral; ' $a$ ', ' $b$ ' and $k d$ are the parameters estimated from Eqn (1); and $k p$ is passage rate, considered in the current study as 0.05.

The NLIN procedure of SAS (SAS Institute Inc., Cary, NC, USA) was used to estimate the parameters from the models. Pearson correlations between analysed contents in feedstuffs and ruminal TA and effective mineral release were performed (CORR procedure; SAS Institute Inc., Cary, NC, USA). All statistical procedures were conducted using 0.05 as the critical level for the probability for the type I error.

Additionally, to identify concentrate and forage sub-groups of mineral release, a cluster analysis was performed using ' $a$ ', ' $b$ ' and $k d$ estimates for TA as response variable. Non-hierarchical $k$-means clustering method was performed (Johnson \& Wichern 1998; Khattree \& Naik 2000) by using the FASTCLUS procedure of SAS (SAS Institute Inc., Cary, NC, USA). Initially, a maximum of five clusters was established for each clustering procedure. If this procedure created at least one cluster with only one observation (feed), a new clustering was made with a maximum of four clusters. This procedure was repeated until each and every cluster had at least two observations (feeds). The efficiency of clustering was also evaluated through the over-all $R^{2}$ and the CCC (cubic clustering criterion).

\section{RESULTS}

Substantial variability was observed between concentrate and forage feedstuffs for all analysed constituents (Table 1). For certain feeds in the current study, the model proposed by Ørskov \& McDonald (1979) did not converge due to different degradation responses. In this case, the model was not adopted because a negative slope was observed; indicative of a greater mineral amount in the residual than in the incubated sample. Calculated parameters of the model, ' $a$ ', ' $b$ ' and $k d$, for concentrate and forage feedstuffs are presented in Tables 2 and 3, respectively. Substantial variability was observed for the effective ruminal degradation and total and individual mineral release (Table 4). When feedstuffs were clustered according with the ' $a$ ', ' $b$ ' and $k d$ estimates of TA ruminal release, four groups were identified (Table 5). From group ' 1 ' to group ' 4 ', an increase in the fraction ' $a$ ', and a reduction in both fraction ' $b$ ' and release rate was observed. A negative correlation was observed between NDF content and mineral release (Table 6). In general, the solubility values for DM, TA and each mineral for majority of feedstuffs ranged between $0 \cdot 6-0 \cdot 8,0 \cdot 7-0 \cdot 9$ and $0 \cdot 9-1 \cdot 0$, respectively, except for $\mathrm{Cu}, \mathrm{Co}$ and Fe (Table 7).

\section{DISCUSSION}

Mineral contents

Reports regarding the availability of minerals from feedstuffs are important to improve the precision of diet formulation and mineral supplementation for cattle. Moreover, mineral content of feedstuffs is an important factor for maintaining buffering capacity in ruminants (Jasaitis et al. 1987). Research in tropical countries has focused primarily on investigating the impact of protein and energy supplementation on cattle performance (Corah 1996; Sath et al. 2012). Furthermore, trace element content is extremely variable in feedstuffs when compared with CP or NDF content (Adams 1975; Playne et al. 1978; Genther \& Hansen 2014). In the current study, while the coefficients of variation (CV) for CP and NDF in forage group were 35.4 and $15 \cdot 2 \%$, respectively, differences between mineral contents were even greater. The average coefficient of variation for minerals in forages was 82.6 and $99 \cdot 7 \%$ when all groups were considered. The smallest CV between minerals was $54 \cdot 1 \%$ for $\mathrm{K}$ and the greatest CV was $133 \cdot 3 \%$ for Fe. 
Table 1. Chemical composition of concentrate and forage feedstuffs

\begin{tabular}{|c|c|c|c|c|c|c|c|c|c|c|c|c|c|c|c|}
\hline \multirow[b]{2}{*}{ Feeds } & \multirow{2}{*}{$\begin{array}{l}\mathrm{DM}^{*} \\
\text { (g/kg) }\end{array}$} & $\mathrm{Ash}^{+}$ & $C P^{\neq}$ & $\mathrm{NDF}^{\S}$ & $\mathrm{Ca}$ & $P$ & $\mathrm{Na}$ & $\mathrm{K}$ & Mg & S & $\mathrm{Cu}$ & $\mathrm{Zn}$ & Mn & $\mathrm{Co}$ & $\mathrm{Fe}$ \\
\hline & & \multicolumn{9}{|c|}{ (g/kg DM) } & \multicolumn{5}{|c|}{ (mg/kg) } \\
\hline \multicolumn{16}{|l|}{ Energy concentrates } \\
\hline Ground ear maize & 875 & 45 & 146 & 588 & 0.9 & $2 \cdot 0$ & $0 \cdot 3$ & $2 \cdot 5$ & 0.9 & $0 \cdot 2$ & $6 \cdot 7$ & 40 & $11 \cdot 9$ & $0 \cdot 37$ & 167 \\
\hline Ground maize & 865 & 13 & 96 & 56 & $0 \cdot 3$ & $2 \cdot 0$ & $0 \cdot 3$ & $3 \cdot 4$ & $1 \cdot 3$ & $0 \cdot 3$ & $2 \cdot 2$ & 12 & $3 \cdot 6$ & $0 \cdot 02$ & 18 \\
\hline Ground sorghum & 863 & 13 & 104 & 66 & $3 \cdot 6$ & $2 \cdot 8$ & $1 \cdot 2$ & 10 & $1 \cdot 8$ & $0 \cdot 3$ & $2 \cdot 4$ & 17 & 10 & $0 \cdot 04$ & 42 \\
\hline Rice bran & 865 & 96 & 164 & 179 & $1 \cdot 1$ & $7 \cdot 7$ & $0 \cdot 2$ & 11 & $9 \cdot 1$ & $1 \cdot 8$ & $5 \cdot 5$ & 56 & 192 & $0 \cdot 25$ & 131 \\
\hline Soybean hulls & 875 & 45 & 146 & 588 & $3 \cdot 8$ & $1 \cdot 2$ & $1 \cdot 2$ & 13 & $3 \cdot 2$ & $0 \cdot 8$ & $7 \cdot 9$ & 31 & $9 \cdot 0$ & $0 \cdot 07$ & 307 \\
\hline Wheat bran & 869 & 49 & 198 & 303 & $1 \cdot 7$ & $8 \cdot 0$ & $1 \cdot 1$ & 12 & $3 \cdot 8$ & $1 \cdot 0$ & 15 & 97 & 153 & $0 \cdot 08$ & 125 \\
\hline \multicolumn{16}{|l|}{ Protein concentrates } \\
\hline Maize gluten & 918 & 44 & 656 & 165 & $0 \cdot 5$ & $3 \cdot 1$ & $0 \cdot 3$ & $2 \cdot 0$ & $1 \cdot 0$ & $4 \cdot 0$ & 23 & 50 & 25 & $0 \cdot 23$ & 153 \\
\hline Cottonseed meal & 891 & 66 & 413 & 192 & $2 \cdot 6$ & $9 \cdot 3$ & $0 \cdot 3$ & 12 & $6 \cdot 1$ & $1 \cdot 0$ & 26 & 63 & 17 & $0 \cdot 21$ & 123 \\
\hline Ground bean & 879 & 63 & 525 & 186 & $3 \cdot 0$ & $5 \cdot 9$ & $0 \cdot 7$ & 17 & $5 \cdot 5$ & $0 \cdot 9$ & 14 & 43 & 26 & $0 \cdot 33$ & 263 \\
\hline Peanut meal & 882 & 38 & 521 & 103 & $1 \cdot 9$ & $6 \cdot 2$ & $0 \cdot 2$ & $9 \cdot 9$ & $4 \cdot 1$ & $0 \cdot 4$ & 22 & 60 & 55 & $0 \cdot 51$ & 691 \\
\hline Soybean meal & 935 & 49 & 465 & 150 & $4 \cdot 3$ & $7 \cdot 6$ & $0 \cdot 7$ & 20 & $3 \cdot 9$ & $1 \cdot 5$ & 18 & 63 & 42 & $0 \cdot 33$ & 247 \\
\hline Sunflower meal & 851 & 63 & 322 & 451 & $3 \cdot 4$ & $8 \cdot 4$ & $1 \cdot 0$ & 11 & $6 \cdot 2$ & $0 \cdot 5$ & 31 & 90 & 36 & $0 \cdot 30$ & 176 \\
\hline \multicolumn{16}{|l|}{ Forages** } \\
\hline Maize silage & 221 & 71 & 67 & 520 & $2 \cdot 5$ & $1 \cdot 6$ & $1 \cdot 1$ & $9 \cdot 1$ & $1 \cdot 6$ & $0 \cdot 8$ & $4 \cdot 6$ & 30 & 27 & $0 \cdot 49$ & 1244 \\
\hline Decumbens & 194 & 87 & 115 & 607 & $5 \cdot 0$ & $0 \cdot 9$ & $0 \cdot 1$ & $9 \cdot 4$ & $0 \cdot 9$ & $0 \cdot 4$ & $3 \cdot 8$ & 28 & 85 & $0 \cdot 28$ & 470 \\
\hline Elephant grass & 237 & 127 & 205 & 528 & $7 \cdot 3$ & $2 \cdot 1$ & $0 \cdot 2$ & 21 & $1 \cdot 8$ & $0 \cdot 9$ & $7 \cdot 2$ & 20 & 81 & $0 \cdot 17$ & 118 \\
\hline Humidicola & 179 & 90 & 105 & 714 & $6 \cdot 0$ & $0 \cdot 9$ & $0 \cdot 2$ & 19 & $1 \cdot 3$ & $0 \cdot 4$ & $3 \cdot 7$ & 19 & 71 & $0 \cdot 09$ & 151 \\
\hline Marandu & 179 & 81 & 107 & 666 & $4 \cdot 2$ & $0 \cdot 9$ & $0 \cdot 2$ & $6 \cdot 8$ & $0 \cdot 9$ & $0 \cdot 3$ & 15 & 10 & 56 & $0 \cdot 46$ & 157 \\
\hline MG-4 & 185 & 72 & 117 & 685 & $4 \cdot 0$ & $0 \cdot 9$ & $0 \cdot 1$ & $5 \cdot 4$ & $0 \cdot 9$ & $0 \cdot 3$ & $2 \cdot 9$ & 19 & 68 & $0 \cdot 09$ & 70 \\
\hline Mombaça & 242 & 108 & 146 & 659 & 14 & $1 \cdot 8$ & $0 \cdot 1$ & 27 & $1 \cdot 5$ & $1 \cdot 2$ & 11 & 34 & 192 & $0 \cdot 41$ & 137 \\
\hline Mulato & 170 & 97 & 88 & 622 & $5 \cdot 1$ & $0 \cdot 9$ & $0 \cdot 1$ & $8 \cdot 1$ & $0 \cdot 9$ & $0 \cdot 2$ & $2 \cdot 1$ & 15 & 38 & $0 \cdot 07$ & 88 \\
\hline Peanut forage & 269 & 77 & 207 & 412 & 24 & $2 \cdot 1$ & $0 \cdot 2$ & $7 \cdot 2$ & $2 \cdot 8$ & $1 \cdot 2$ & 14 & 29 & 162 & $0 \cdot 48$ & 83 \\
\hline Tanzania & 236 & 91 & 142 & 607 & $7 \cdot 8$ & $1 \cdot 1$ & $0 \cdot 1$ & 15 & $1 \cdot 2$ & $0 \cdot 9$ & $6 \cdot 3$ & 22 & 51 & $0 \cdot 23$ & 54 \\
\hline Tifton 85 hay & 868 & 50 & 87 & 753 & $3 \cdot 3$ & $1 \cdot 6$ & $0 \cdot 1$ & 12 & $0 \cdot 9$ & $2 \cdot 0$ & 21 & 79 & 91 & $0 \cdot 13$ & 92 \\
\hline Xaraes & 209 & 79 & 106 & 644 & $6 \cdot 0$ & $0 \cdot 9$ & $0 \cdot 1$ & $6 \cdot 9$ & $1 \cdot 1$ & $0 \cdot 3$ & $4 \cdot 2$ & 22. & 63 & $0 \cdot 13$ & 72 \\
\hline
\end{tabular}

* Dry matter.

+ Total mineral content.

₹ Crude protein.

$\S$ Neutral detergent fibre.

** The forages: Decumbens grass (Urochloa decumbens), Elephant grass (Pennisetum purpureum), Humidicola grass (Urochloa humidicola), Marandu grass (Urochloa brizantha cv Marandu), MG-4 grass (Urochloa brizantha cv MG-4), Mombaça grass (Panicum maximum cv Mombaça), Mulato grass (hybrid Urochloa decumbens $\times$ Urochloa ruziziensis), Peanut forage (Arachis pintoi), Tanzania grass (Panicum maximum cv Tanzânia), Tifton 85 hay (Cynodon spp.) and Xaraes grass (Urochloa brizantha cv Xaraés) were collected after $45 \mathrm{~d}$ of regrowth.

Feedstuff $\mathrm{Ca}, \mathrm{Mg}, \mathrm{K}, \mathrm{Cl}$ and $\mathrm{Fe}$ contents are in appropriate concentrations to meet the mineral requirements of beef cattle (Minson 1990; Chládek \& Zapletal 2007). Smart et al. (1981), in a review about the interactions across $\mathrm{Cu}, \mathrm{Zn}$ and $\mathrm{Se}$ in soil, plants and cattle, reported that basal feedstuffs can provide trace minerals to the animal, however, concentrations can be inadequate. Thus, Yoshihara et al. (2013) estimated mineral intake and nutritional quality for grazed cattle varying numbers and combinations of 17 temperate pasture species; they suggested that by improving the number of plant species or products in diet formulation or in grazed pastures, improvement in mineral balance can be obtained in cattle.

Rumen mineral release

Mineral requirements from ruminal fauna are not negligible (Bravo et al. 2000), consequently mineral release from ingested feeds is important. Although mineral solubilization does not guarantee mineral 
Table 2. Dry matter and total and individual mineral parameters of ruminal release of concentrate feeds

\begin{tabular}{|c|c|c|c|c|c|c|c|c|c|c|c|c|c|c|}
\hline Feeds & Parameter* & $\mathrm{DM}^{+}$ & $\mathrm{Ash}^{*}$ & $\mathrm{Ca}$ & $\mathrm{P}$ & $\mathrm{Na}$ & K & $M g$ & $S$ & $\mathrm{Cu}$ & $\mathrm{Zn}$ & Mn & Co & $\mathrm{Fe}$ \\
\hline \multirow[t]{3}{*}{ Ground ear maize } & $a$ & 10 & 46 & $9 \cdot 0$ & $6 \cdot 1$ & $8 \cdot 4$ & $5 \cdot 7$ & $8 \cdot 1$ & $6 \cdot 9$ & 43 & 12 & $4 \cdot 6$ & $7 \cdot 1$ & $8 \cdot 1$ \\
\hline & $b$ & 71 & 41 & 46 & 83 & 67 & 92 & 69 & 49 & 51 & 62 & 56 & 80 & 69 \\
\hline & $K d$ & $5 \cdot 0$ & 14 & $7 \cdot 0$ & 73 & 43 & 171 & 36 & $7 \cdot 0$ & $2 \cdot 0$ & 25 & $7 \cdot 0$ & $65 \cdot 0$ & 36 \\
\hline \multirow[t]{3}{*}{ Ground maize } & $a$ & 18 & 52 & $9 \cdot 0$ & 12 & 11 & 65 & 73 & 11 & 32 & 18 & $8 \cdot 4$ & 11 & 13 \\
\hline & $b$ & 80 & 43 & 95 & 76 & 83 & 34 & 22 & 77 & 70 & 79 & 98 & 88 & 71 \\
\hline & $K d$ & $6 \cdot 0$ & $7 \cdot 0$ & $1 \cdot 0$ & 63 & 137 & 169 & 47 & 232 & $3 \cdot 0$ & $5 \cdot 0$ & $2 \cdot 0$ & 218 & 47 \\
\hline \multirow[t]{3}{*}{ Ground sorghum } & $a$ & 18 & 18 & $7 \cdot 3$ & 69 & $3 \cdot 7$ & $9 \cdot 2$ & $1 \cdot 0$ & 10 & $7 \cdot 7$ & 29 & 10 & _\$ & $9 \cdot 3$ \\
\hline & $b$ & 78 & 68 & 85 & 24 & 97 & 90 & 99 & 82 & 54 & 55 & 75 & - & 82 \\
\hline & $K d$ & $5 \cdot 0$ & 16 & 20 & 152 & 17 & 147 & 17 & 22 & 51 & $5 \cdot 0$ & 60 & - & 91 \\
\hline \multirow[t]{3}{*}{ Rice bran } & $a$ & 11 & 17 & 67 & $8 \cdot 0$ & $8 \cdot 8$ & 48 & 83 & $8 \cdot 9$ & $8 \cdot 9$ & 12 & 10 & $8 \cdot 9$ & $9 \cdot 1$ \\
\hline & $b$ & 55 & 45 & 30 & 64 & 90 & 49 & 10 & 64 & 64 & 64 & 72 & 65 & 84 \\
\hline & $K d$ & 88 & 88 & 45 & 21 & 189 & 86 & 78 & 105 & 105 & 40 & 61 & 86 & 86 \\
\hline \multirow[t]{3}{*}{ Soybean hulls } & $a$ & 13 & 18 & 23 & 24 & $4 \cdot 8$ & 27 & 48 & 75 & $5 \cdot 6$ & 21 & 20 & $8 \cdot 9$ & $7 \cdot 7$ \\
\hline & $b$ & 80 & 71 & 70 & 62 & 94 & 72 & 57 & 33 & 66 & 52 & 68 & 84 & 97 \\
\hline & $K d$ & $4 \cdot 0$ & 20 & $3 \cdot 0$ & 97 & $8 \cdot 0$ & 149 & $3 \cdot 0$ & $1 \cdot 0$ & 100 & $9 \cdot 0$ & $4 \cdot 0$ & 38 & $3 \cdot 0$ \\
\hline \multirow[t]{3}{*}{ Wheat bran } & $a$ & 11 & $0 \cdot 3$ & 17 & 75 & 88 & 10 & 16 & - & 12 & $8 \cdot 1$ & 11 & 10 & 10 \\
\hline & $b$ & 64 & 89 & 43 & 25 & 8 & 90 & 76 & - & 80 & 84 & 85 & 34 & 81 \\
\hline & $K d$ & 36 & 51 & 17 & 24 & 14 & 156 & 38 & - & 29 & 20 & 18 & 21 & 38 \\
\hline \multirow[t]{3}{*}{ Maize gluten } & $a$ & $9 \cdot 2$ & $0 \cdot 1$ & $1 \cdot 5$ & 11 & $0 \cdot 2$ & 61 & 81 & - & 19 & $3 \cdot 0$ & $3 \cdot 0$ & $1 \cdot 7$ & $1 \cdot 7$ \\
\hline & $b$ & 54 & 90 & 34 & 74 & 85 & 37 & 12 & - & 58 & 56 & 56 & 91 & 91 \\
\hline & $K d$ & $3 \cdot 0$ & 23 & 103 & 68 & 49 & 220 & 10 & - & $3 \cdot 0$ & 11 & 11 & 77 & 77 \\
\hline \multirow[t]{3}{*}{ Cottonseed meal } & $a$ & 19 & 28 & 25 & 16 & - & 16 & 17 & 61 & 17 & 14 & - & 15 & 16 \\
\hline & $b$ & 52 & 62 & 50 & 81 & - & 80 & 70 & 15 & 72 & 68 & - & 33 & 71 \\
\hline & $K d$ & 14 & 46 & $8 \cdot 0$ & 40 & - & 100 & 31 & 191 & 35 & 14 & - & 100 & 31 \\
\hline \multirow[t]{3}{*}{ Ground bean } & $a$ & 15 & 11 & 43 & $9 \cdot 0$ & $8 \cdot 1$ & $8 \cdot 9$ & 64 & $9 \cdot 1$ & 12 & 13 & $9 \cdot 4$ & 16 & 14 \\
\hline & $b$ & 78 & 82 & 49 & 89 & 86 & 91 & 31 & 83 & 82 & 78 & 87 & 68 & 74 \\
\hline & $K d$ & 14 & 77 & $5 \cdot 0$ & 83 & 13 & 162 & 24 & 93 & 24 & 29 & $2 \cdot 0$ & 26 & 24 \\
\hline \multirow[t]{3}{*}{ Peanut meal } & a & 23 & $1 \cdot 0$ & $5 \cdot 5$ & 17 & $0 \cdot 8$ & 17 & 18 & 19 & 21 & 19 & 17 & 25 & 18 \\
\hline & $b$ & 64 & 96 & 80 & 79 & 86 & 82 & 76 & 63 & 69 & 61 & 76 & 59 & 76 \\
\hline & $K d$ & 15 & 16 & $8 \cdot 0$ & 78 & $8 \cdot 0$ & 167 & 46 & 34 & 31 & 38 & $8 \cdot 0$ & 11 & 46 \\
\hline \multirow[t]{3}{*}{ Soybean meal } & a & 15 & $0 \cdot 5$ & $8 \cdot 6$ & 11 & $9 \cdot 1$ & 14 & 12 & 55 & 16 & $6 \cdot 7$ & 13 & 17 & 11 \\
\hline & $b$ & 80 & 99 & 88 & 87 & 89 & 86 & 85 & 42 & 80 & 93 & 85 & 75 & 86 \\
\hline & $K d$ & 12 & 87 & 10 & 40 & 21 & 178 & 27 & 59 & $9 \cdot 0$ & $6 \cdot 0$ & $7 \cdot 0$ & 16 & 27 \\
\hline \multirow[t]{3}{*}{ Sunflower meal } & $a$ & 10 & 27 & $8 \cdot 9$ & 10 & 55 & $6 \cdot 4$ & 19 & $8 \cdot 0$ & 10 & $7 \cdot 0$ & $1 \cdot 0$ & $6 \cdot 4$ & $8 \cdot 2$ \\
\hline & $b$ & 52 & 66 & 70 & 87 & 36 & 92 & 74 & 58 & 76 & 64 & 85 & 51 & 85 \\
\hline & $K d$ & 21 & 57 & 11 & 49 & 22 & 122 & 36 & 22 & 31 & 36 & $8 \cdot 0$ & 125 & 36 \\
\hline
\end{tabular}

* ' $a$ ' is the immediately soluble fraction; ' $b$ ' is the insoluble by potentially degradable/releasable fraction; and $k d$ is degradation/release rate of ' $b$ ' $(\% / h)$.

+ Dry matter.

\# Total mineral content.

$\S(-)$ means that the model proposed by Ørskov \& McDonald (1979) did not converge due to different release response of minerals.

utilization, absorption, or tissue deposition, absorption is largely affected by mineral intake and mineral solubilization in the gastrointestinal tract prior to absorption (Field 1981; Flachowsky et al. 1994). Like this, Van Eys \& Reid (1987) have reported that reduced mineral release from forages decreases performance in grazing cattle.
High variability in mineral content between feedstuffs, and different solubilities between minerals have been described (Čerešňáková et al. 2007). In the current study, high variability was observed for effective release among minerals and effective release in each mineral between feeds. Feeds with low DM degradation did not necessarily have low 
Table 3. DM and total and individual mineral parameters of ruminal release of forage feeds

\begin{tabular}{|c|c|c|c|c|c|c|c|c|c|c|c|c|c|c|}
\hline Feeds & Parameter* & $\mathrm{DM}^{+}$ & $\mathrm{Ash}^{\ddagger}$ & $\mathrm{Ca}$ & $\mathrm{P}$ & $\mathrm{Na}$ & $\mathrm{K}$ & $\mathrm{Mg}$ & $\mathrm{S}$ & $\mathrm{Cu}$ & $\mathrm{Zn}$ & Mn & Co & $\mathrm{Fe}$ \\
\hline \multirow[t]{3}{*}{ Maize silage } & $a$ & 39 & 56 & 34 & 79 & $-^{\S}$ & - & 76 & 77 & 38 & 40 & 36 & 45 & 36 \\
\hline & $b$ & 42 & 20 & 35 & $9 \cdot 1$ & - & - & 12 & 18 & 37 & 41 & 32 & 52 & 31 \\
\hline & $K d$ & $4 \cdot 0$ & $3 \cdot 0$ & $9 \cdot 0$ & $2 \cdot 0$ & - & - & $9 \cdot 0$ & $4 \cdot 0$ & 18 & 29 & 18 & $1 \cdot 0$ & $9 \cdot 0$ \\
\hline \multirow[t]{3}{*}{ Decumbens } & a & 21 & 67 & 17 & - & - & - & 23 & $9 \cdot 3$ & 19 & 22 & 25 & 12 & 23 \\
\hline & $b$ & 63 & 14 & 67 & - & - & - & 58 & 78 & 41 & 46 & 66 & 60 & 58 \\
\hline & $K d$ & $5 \cdot 0$ & 24 & $8 \cdot 0$ & - & - & - & 16 & $7 \cdot 0$ & $6 \cdot 0$ & 11 & 29 & 4 & 16 \\
\hline \multirow[t]{3}{*}{ Elephant grass } & $a$ & 16 & 38 & 10 & 57 & 65 & 99 & 50 & 69 & 11 & 16 & 18 & 17 & 17 \\
\hline & $b$ & 61 & 26 & 79 & 18 & 25 & $0 \cdot 5$ & 42 & 25 & 61 & 30 & 74 & 57 & 70 \\
\hline & $K d$ & $5 \cdot 0$ & $9 \cdot 0$ & $7 \cdot 0$ & 62 & $5 \cdot 0$ & 32 & 10 & $4 \cdot 0$ & $4 \cdot 0$ & $15 \cdot 0$ & 12 & $0 \cdot 1$ & 10 \\
\hline \multirow[t]{3}{*}{ Humidicola } & $a$ & 18 & 54 & 18 & - & 22 & 99 & 34 & 22 & $8 \cdot 3$ & 18 & 20 & 12 & 21 \\
\hline & $b$ & 59 & 19 & 65 & - & 50 & $0 \cdot 7$ & 53 & 51 & 49 & 33 & 69 & 85 & 63 \\
\hline & $K d$ & $5 \cdot 0$ & 11 & $8 \cdot 0$ & - & 20 & 29 & 26 & $2 \cdot 0$ & 10 & 14 & 33 & $6 \cdot 0$ & 26 \\
\hline \multirow[t]{3}{*}{ Marandu } & $a$ & 19 & 69 & 16 & - & 64 & 98 & 22 & 22 & 18 & $7 \cdot 0$ & 22 & 38 & 22 \\
\hline & $b$ & 64 & 11 & 66 & - & 23 & $1 \cdot 9$ & 57 & 63 & 42 & 28 & 64 & 40 & 57 \\
\hline & $K d$ & $5 \cdot 0$ & 10 & $8 \cdot 0$ & - & $9 \cdot 0$ & 168 & 19 & $5 \cdot 0$ & $9 \cdot 0$ & 10 & $34 \cdot 0$ & $4 \cdot 0$ & 19 \\
\hline \multirow[t]{3}{*}{ MG-4 } & a & 21 & 68 & 16 & - & - & 98 & 24 & 18 & $6 \cdot 4$ & 18 & 24 & 25 & 24 \\
\hline & $b$ & 63 & $9 \cdot 1$ & 68 & - & - & $1 \cdot 2$ & 58 & 71 & 51 & 52 & 66 & 58 & 58 \\
\hline & $K d$ & $5 \cdot 0$ & 23 & $8 \cdot 0$ & - & - & 56 & 18 & $0 \cdot 1$ & $8 \cdot 0$ & 13 & 27 & $8 \cdot 0$ & 18 \\
\hline \multirow[t]{3}{*}{ Mombaça } & $a$ & 17 & 44 & 29 & - & 25 & 99 & 22 & 41 & 31 & - & 20 & 18 & 22 \\
\hline & $b$ & 56 & 28 & 55 & - & 40 & $0 \cdot 7$ & 57 & 45 & 44 & - & 58 & 53 & 57 \\
\hline & $K d$ & $4 \cdot 0$ & $5 \cdot 0$ & 10 & - & 13 & 60 & 30 & $0 \cdot 0$ & $11 \cdot 0$ & - & 42 & $9 \cdot 0$ & 30 \\
\hline \multirow[t]{3}{*}{ Mulato } & a & 22 & 65 & 19 & - & 22 & 99 & 23 & $6 \cdot 8$ & 21 & 19 & 22 & 11 & 23 \\
\hline & $b$ & 60 & 12 & 63 & - & 55 & $0 \cdot 8$ & 60 & 71 & 56 & 38 & 62 & 80 & 60 \\
\hline & $K d$ & $5 \cdot 0$ & $5 \cdot 0$ & 11 & - & $0 \cdot 1$ & 27 & 17 & $6 \cdot 0$ & 20 & 17 & $0 \cdot 2$ & 16 & 17 \\
\hline \multirow[t]{3}{*}{ Peanut forage } & a & 28 & 42 & 34 & 41 & 18 & 97 & 16 & 34 & 21 & 26 & 28 & 17 & 16 \\
\hline & $b$ & 56 & 43 & 49 & 22 & 70 & $2 \cdot 4$ & 75 & 42 & 49 & 38 & 59 & 68 & 75 \\
\hline & $K d$ & $8 \cdot 0$ & 12 & 14 & 32 & 11 & 32 & $8 \cdot 0$ & 20 & $7 \cdot 0$ & 12 & 17 & 10 & $8 \cdot 0$ \\
\hline \multirow[t]{3}{*}{ Tanzania } & $a$ & 20 & 46 & 16 & - & 16 & 95 & 47 & 27 & 23 & 20 & 25 & $8 \cdot 5$ & 23 \\
\hline & $b$ & 57 & 25 & 63 & - & 27 & $4 \cdot 6$ & 34 & 61 & 30 & 29 & 39 & 26 & 50 \\
\hline & $K d$ & $4 \cdot 0$ & $8 \cdot 0$ & $8 \cdot 0$ & - & $10 \cdot 0$ & 166 & $6 \cdot 0$ & $4 \cdot 0$ & $5 \cdot 0$ & $7 \cdot 0$ & $12 \cdot 0$ & $7 \cdot 0$ & $6 \cdot 0$ \\
\hline \multirow[t]{3}{*}{ Tifton 85 hay } & $a$ & 12 & 62 & 13 & - & 10 & 98 & 22 & 87 & $2 \cdot 3$ & $5 \cdot 3$ & 12 & $3 \cdot 0$ & 13 \\
\hline & $b$ & 53 & $9 \cdot 3$ & 13 & - & 64 & $1 \cdot 3$ & 34 & $7 \cdot 3$ & 28 & 42 & 58 & 37 & 38 \\
\hline & $K d$ & $4 \cdot 0$ & $4 \cdot 0$ & 191 & - & $6 \cdot 0$ & 39 & 18 & 10 & $7 \cdot 0$ & $6 \cdot 0$ & 21 & 28 & 18 \\
\hline \multirow[t]{3}{*}{ Xaraes } & $a$ & 18 & 62 & 12 & - & 20 & 98 & 22 & 8 & $4 \cdot 8$ & 11 & 21 & 23 & 22 \\
\hline & $b$ & 65 & 15 & 74 & - & 45 & $2 \cdot 0$ & 60 & 82 & 58 & 61 & 67 & 61 & 60 \\
\hline & $K d$ & $4 \cdot 0$ & $9 \cdot 0$ & $8 \cdot 0$ & - & 19 & 34 & 20 & $4 \cdot 0$ & $8 \cdot 0$ & 13 & 21 & 22 & 20 \\
\hline
\end{tabular}

* ' $a$ ' is the immediately soluble fraction; ' $b$ ' is the insoluble by potentially degradable/releasable fraction; and $k d$ is degradation/releasing rate of ' $b$ ' $(\% / h)$.

+ Dry matter.

₹ Total mineral content.

$\S(-)$ means that the model proposed by Ørskov \& McDonald (1979) did not converge due to different release response of minerals.

mineral release coefficients (Emanuele et al. 1991). Mineral release profiles in relationship to DM digestibility can be associated with concentration of minerals in the digestible part of the plant. Most minerals are associated with the organic constituents within feeds (Broadley et al. 2007; Maathuis \& Diatloff 2012; He et al. 2014). This is most likely the reason for the lack of a relationship between DM digestibility and mineral solubility.

The average effective release of $\mathrm{K}$ was 0.98 for concentrates and forages, being the lowest variation measured among minerals. Potassium in the plant cell is 
Table 4. Coefficients of DM rumen effective degradation, and total and individual mineral rumen release in concentrate and forage feeds considering passage rate $=0 \cdot 05 / \mathrm{h}$

\begin{tabular}{|c|c|c|c|c|c|c|c|c|c|c|c|c|c|}
\hline Feeds & $\mathrm{DM}^{*}$ & $\mathrm{Ash}^{+}$ & $\mathrm{Ca}$ & $P$ & $\mathrm{Na}$ & $\mathrm{K}$ & Mg & $\mathrm{S}$ & $\mathrm{Cu}$ & $\mathrm{Zn}$ & $\mathrm{Mn}$ & Co & $\mathrm{Fe}$ \\
\hline \multicolumn{14}{|l|}{ Concentrates } \\
\hline Ground ear maize & $0 \cdot 45$ & $0 \cdot 76$ & $0 \cdot 36$ & $0 \cdot 84$ & $0 \cdot 68$ & $0 \cdot 95$ & $0 \cdot 69$ & $0 \cdot 35$ & 0.57 & $0 \cdot 64$ & $0 \cdot 37$ & $0 \cdot 81$ & $0 \cdot 69$ \\
\hline Ground maize & $0 \cdot 61$ & $0 \cdot 77$ & $0 \cdot 30$ & $0 \cdot 82$ & 0.91 & 0.98 & 0.93 & $0 \cdot 86$ & 0.59 & 0.58 & $0 \cdot 36$ & 0.97 & $0 \cdot 76$ \\
\hline Ground sorghum & $0 \cdot 57$ & $0 \cdot 70$ & 0.75 & 0.92 & 0.79 & 0.96 & $0 \cdot 78$ & $0 \cdot 76$ & 0.57 & 0.57 & 0.79 & ${ }_{-}^{\ddagger}$ & $0 \cdot 87$ \\
\hline Rice bran & $0 \cdot 63$ & $0 \cdot 60$ & 0.94 & $0 \cdot 59$ & 0.97 & $0 \cdot 93$ & 0.93 & $0 \cdot 70$ & $0 \cdot 70$ & $0 \cdot 69$ & 0.76 & $0 \cdot 70$ & $0 \cdot 89$ \\
\hline Soybean hulls & $0 \cdot 48$ & $0 \cdot 74$ & $0 \cdot 48$ & $0 \cdot 83$ & $0 \cdot 62$ & $0 \cdot 96$ & $0 \cdot 68$ & $0 \cdot 81$ & $0 \cdot 68$ & $0 \cdot 54$ & $0 \cdot 50$ & $0 \cdot 83$ & $0 \cdot 41$ \\
\hline Wheat bran & $0 \cdot 67$ & $0 \cdot 81$ & $0 \cdot 51$ & $0 \cdot 95$ & 0.94 & $0 \cdot 97$ & $0 \cdot 83$ & - & $0 \cdot 80$ & 0.75 & 0.77 & $0 \cdot 38$ & $0 \cdot 82$ \\
\hline Maize gluten & $0 \cdot 29$ & $0 \cdot 74$ & $0 \cdot 34$ & $0 \cdot 80$ & 0.77 & $0 \cdot 98$ & $0 \cdot 89$ & - & $0 \cdot 38$ & $0 \cdot 42$ & $0 \cdot 42$ & $0 \cdot 87$ & $0 \cdot 87$ \\
\hline Cottonseed meal & $0 \cdot 58$ & $0 \cdot 84$ & $0 \cdot 56$ & $0 \cdot 88$ & - & $0 \cdot 92$ & $0 \cdot 77$ & $0 \cdot 75$ & $0 \cdot 80$ & $0 \cdot 64$ & - & $0 \cdot 46$ & $0 \cdot 77$ \\
\hline Ground bean & 0.72 & $0 \cdot 88$ & $0 \cdot 67$ & $0 \cdot 93$ & $0 \cdot 71$ & $0 \cdot 97$ & 0.90 & $0 \cdot 88$ & $0 \cdot 80$ & $0 \cdot 80$ & $0 \cdot 30$ & 0.73 & $0 \cdot 75$ \\
\hline Peanut meal & $0 \cdot 71$ & $0 \cdot 74$ & $0 \cdot 54$ & $0 \cdot 92$ & $0 \cdot 54$ & $0 \cdot 97$ & $0 \cdot 87$ & $0 \cdot 73$ & $0 \cdot 80$ & 0.73 & 0.63 & 0.65 & $0 \cdot 87$ \\
\hline Soybean meal & $0 \cdot 71$ & $0 \cdot 94$ & $0 \cdot 67$ & $0 \cdot 88$ & $0 \cdot 81$ & $0 \cdot 97$ & $0 \cdot 84$ & 0.93 & $0 \cdot 67$ & 0.59 & $0 \cdot 62$ & $0 \cdot 74$ & $0 \cdot 84$ \\
\hline Sunflower meal & $0 \cdot 52$ & $0 \cdot 87$ & $0 \cdot 57$ & $0 \cdot 89$ & $0 \cdot 84$ & $0 \cdot 95$ & $0 \cdot 85$ & $0 \cdot 55$ & $0 \cdot 75$ & 0.63 & $0 \cdot 54$ & $0 \cdot 55$ & $0 \cdot 83$ \\
\hline \multicolumn{14}{|l|}{ Forages } \\
\hline Maize silage & $0 \cdot 57$ & $0 \cdot 63$ & $0 \cdot 56$ & $0 \cdot 82$ & - & - & $0 \cdot 83$ & $0 \cdot 84$ & 0.67 & 0.75 & $0 \cdot 61$ & $0 \cdot 52$ & $0 \cdot 56$ \\
\hline Decumbens & 0.52 & $0 \cdot 78$ & $0 \cdot 58$ & - & - & - & 0.67 & $0 \cdot 56$ & $0 \cdot 41$ & $0 \cdot 54$ & $0 \cdot 80$ & 0.40 & 0.67 \\
\hline Elephant grass & 0.47 & $0 \cdot 55$ & $0 \cdot 57$ & $0 \cdot 74$ & 0.78 & $1 \cdot 00$ & $0 \cdot 78$ & $0 \cdot 80$ & $0 \cdot 38$ & $0 \cdot 38$ & $0 \cdot 70$ & $0 \cdot 51$ & $0 \cdot 64$ \\
\hline Humidicola & $0 \cdot 47$ & $0 \cdot 66$ & $0 \cdot 57$ & - & 0.62 & $1 \cdot 00$ & $0 \cdot 78$ & $0 \cdot 37$ & $0 \cdot 41$ & 0.42 & $0 \cdot 80$ & $0 \cdot 60$ & $0 \cdot 74$ \\
\hline Marandu & $0 \cdot 51$ & $0 \cdot 76$ & $0 \cdot 57$ & - & 0.79 & 0.99 & 0.67 & $0 \cdot 53$ & $0 \cdot 45$ & $0 \cdot 26$ & 0.77 & $0 \cdot 54$ & 0.67 \\
\hline MG-4 & $0 \cdot 52$ & $0 \cdot 76$ & $0 \cdot 56$ & - & - & 0.99 & 0.69 & $0 \cdot 63$ & $0 \cdot 37$ & 0.55 & $0 \cdot 80$ & 0.59 & $0 \cdot 69$ \\
\hline Mombaça & $0 \cdot 41$ & $0 \cdot 58$ & $0 \cdot 66$ & - & $0 \cdot 54$ & $1 \cdot 00$ & $0 \cdot 71$ & $0 \cdot 57$ & $0 \cdot 60$ & - & 0.71 & $0 \cdot 51$ & $0 \cdot 71$ \\
\hline Mulato & $0 \cdot 53$ & $0 \cdot 71$ & $0 \cdot 63$ & - & 0.61 & 0.99 & 0.69 & $0 \cdot 46$ & $0 \cdot 66$ & 0.48 & 0.71 & 0.72 & $0 \cdot 69$ \\
\hline Peanut forage & $0 \cdot 61$ & $0 \cdot 73$ & $0 \cdot 70$ & $0 \cdot 60$ & 0.66 & 0.99 & 0.62 & $0 \cdot 67$ & $0 \cdot 50$ & 0.53 & 0.73 & 0.63 & $0 \cdot 62$ \\
\hline Tanzania & $0 \cdot 46$ & $0 \cdot 61$ & $0 \cdot 54$ & - & $0 \cdot 34$ & 0.99 & 0.67 & $0 \cdot 55$ & $0 \cdot 38$ & $0 \cdot 37$ & 0.52 & $0 \cdot 24$ & $0 \cdot 51$ \\
\hline Tifton 85 hay & $0 \cdot 35$ & $0 \cdot 66$ & $0 \cdot 26$ & - & $0 \cdot 44$ & $0 \cdot 99$ & 0.49 & $0 \cdot 92$ & $0 \cdot 18$ & $0 \cdot 27$ & 0.59 & $0 \cdot 34$ & $0 \cdot 43$ \\
\hline Xaraes & $0 \cdot 48$ & $0 \cdot 72$ & $0 \cdot 57$ & - & $0 \cdot 55$ & $0 \cdot 99$ & $0 \cdot 70$ & $0 \cdot 46$ & $0 \cdot 40$ & $0 \cdot 56$ & $0 \cdot 88$ & 0.73 & $0 \cdot 70$ \\
\hline
\end{tabular}

* Dry matter.

+ Total mineral content.

₹ (-) means that the model proposed by Ørskov \& McDonald (1979) did not converge due to different release response of minerals.

not associated with organic matter and persists as the $\mathrm{K}^{+}$ion (Amtmann \& Rubio 2012) and this helps to explain the high release of $K$. Phosphorus release was assumed to be similar to that of K. However, $\mathrm{P}$ within the concentrate feeds is primarily associated with phytate. Rumen micro-organisms synthesize a phytase enzyme in significant amounts (Ray et al. 2013) that help to liberate $P$ from the plant cell. Feeds with low $\mathrm{P}$ content were contaminated with $\mathrm{P}$ from rumen micro-organisms and consequently $\mathrm{P}$ concentrations in the residual DM after rumen incubation were greater than the $\mathrm{P}$ content in the intact feeds, which caused $P$ release to have negative values. This behaviour for $\mathrm{P}$ has been related to feeds with low $\mathrm{P}$ content and high NDF content (Bonhomme 1990; Bravo et al. 2000). However, $\mathrm{P}$ contamination was observed at times $>8 \mathrm{~h}$, probably due to the lag time for bacterial growth and adherence to fibre. Phosphorus content in the residual DM of forages submitted to ruminal incubation $(t=8 \mathrm{~h})$ indicates that 0.52 (maximum: maize silage, 0.77 ; minimum: Tanzania, 0.37) of initial $P$ was released, and after this time, the $\mathrm{P}$ content of residue increased.

Group ' 1 ' was characterized by reduced fraction ' $a$ ' $(3 \pm 4 \cdot 9)$, but greater fraction ' $b$ ' and release rate (91 \pm 6.7 and $0 \cdot 5 \pm 0 \cdot 31$, respectively). In contrast, group ' 4 ' was characterized by greater fraction ' $a$ ' $(63 \pm 5 \cdot 7)$, but reduced fraction ' $b$ ' and release rate $(14 \pm 4 \cdot 1$ and $0 \cdot 1$ $\pm 0 \cdot 08$, respectively). Only concentrates assigned to group ' 1 ' were characterized by 'highest release rate': wheat bran, maize gluten, ground bean, peanut meal and soybean meal. This group was 
Table 5. Cluster classification based in soluble ('a'), insoluble but potentially degradable/releasable fraction (' $b$ '); and release rate of ' $b$ ' ( $k d)$ fractions of total ash of feeds

\begin{tabular}{lrll}
\hline \hline Cluster \# & \multicolumn{1}{l}{$a$} & $b$ & $k d$ \\
\hline 1 & $3 \pm 4 \cdot 9$ & $91 \pm 6 \cdot 7$ & $0 \cdot 5 \pm 0 \cdot 31$ \\
2 & $22 \pm 5 \cdot 2$ & $63 \pm 10 \cdot 1$ & $0 \cdot 5 \pm 0 \cdot 29$ \\
3 & $45 \pm 4 \cdot 5$ & $34 \pm 8 \cdot 9$ & $0 \cdot 1 \pm 0 \cdot 03$ \\
4 & $63 \pm 5 \cdot 7$ & $14 \pm 4 \cdot 1$ & $0 \cdot 1 \pm 0 \cdot 08$ \\
\hline \hline
\end{tabular}

Cluster \#1: wheat bran, maize gluten, ground bean, peanut meal, soybean meal; Cluster \#2: ground sorghum, rice bran, soybean hulls, cottonseed meal, sunflower meal; Cluster \#3: ground ear maize, ground maize, elephant grass, Mombaça, peanut forage, tanzania; and Cluster \#4: maize silage, decumbens, humidicola, marandu, MG-4, Mulato, Tifton85 hay, Xaraes.

composed only of concentrate feedstuffs considered as protein feeds, reaffirming a correlation of 0.41 between CP content and TA release. However, across minerals, a positive and significant relationship between CP content and mineral release was observed only for $\mathrm{Mg}$, $\mathrm{Cu}$ and Fe. Furthermore, from these five feeds, four are by-product feeds that were processed during manufacturing (i.e. heat, pressing, substances added). This may be the reason for a high mineral release rate from these feedstuffs. A second group (group ' 2 ') was similarly composed exclusively of concentrate feeds (ground sorghum, rice bran, soybean hulls, cottonseed meal, sunflower meal). These feeds presented a high release rate $(0 \cdot 5 \pm$ $0 \cdot 29)$, like group ' 1 ', but greater fraction ' $a$ ' $(22 \pm$ $5 \cdot 2)$ and lower fraction ' $b$ ' $(63 \pm 10 \cdot 1)$. In contrast, group ' 4 ' was composed exclusively of forages (maize silage, Decumbens, Humidicola, Marandu, MG-4, Mulato, Tifton-85 hay and Xaraes). This could be related to high $\mathrm{K}$ content and solubility in these feeds. Group ' 3 ' was composed of ground ear maize, ground maize, elephant grass, Mombaça, peanut forage and Tanzania, with ' $a$ ' $=45 \pm 4 \cdot 5$, ' $b$ ' $=34 \pm 8 \cdot 9$, and $k d=0.09 \pm 0.032$.

Relations between feed content and ruminal mineral release

Associations between nutrient contents and mineral release within feedstuffs do exist (Emanuele \& Staples 1990). The correlation between NDF content and ruminal TA release was negative $(r=-0 \cdot 39)$, which may be related to the high capacity of NDF to exchange cations (McBurney et al. 1986; Jasaitis et al. 1987). Moreover, minerals associated with the plant cell wall have a lower bioavailability or require a longer fermentation time for maximal release (Emanuele \& Staples 1990; Flachowsky \& Grün 1992). A negative correlation between Ca release and NDF content, for example, can be explained by the function of $\mathrm{Ca}$ in the cell in that it helps to regulate the control of cell wall enzymes, assists with cell wall stabilization and binds with pectin in the cell wall to help support the plant (Demarty et al. 1984; Spears 1996). Furthermore, with the advancement of plant maturity, NDF and silica concentrations are increased (Smith et al. 1971; Jung \& Allen 1995; Spanghero et al. 2015). Silica content was not measured in the current study; however, this relationship may be confirmed when a correlation of 0.57 was observed between NDF and ruminal TA content, and the average correlation between each mineral and NDF contents was $r=$ $-0 \cdot 31$. Among minerals, only $\mathrm{K}$ and $\mathrm{Mn}$ were positively correlated with NDF content $(0.56$ and 0.34 , respectively).

The correlation between TA content and ruminal TA release was negative $(r=-0 \cdot 51)$. Ma \& Yamaji (2006) reported that insoluble silica is incorporated into the plant cell wall to improve cellular protection. However, ruminal release of each mineral was increased by the contents of other nutrients (average of $r=0.35$ ). As minerals occur in feeds in soluble and insoluble forms, a greater concentration of each mineral is associated with the soluble fraction.

\section{Abomasal mineral release}

In general, minerals with nutritional importance have greater solubility as $\mathrm{pH}$ decreases. At a high $\mathrm{pH}$, minerals typically become insoluble, thus decreasing the chances of absorption. The $24 \mathrm{~h}$ ruminal residues had negative mineral release values in some cases. This could be related to contamination from minerals of ruminal fluid origin (Moreira et al. 2013). In comparison with residual samples incubated in the rumen for $24 \mathrm{~h}$, solubility of minerals with nutritional interest was numerically greater than DM and TA. Few reports have been published investigating mineral solubility of different feedstuffs in ruminant animals. It is difficult to explain the reason for the reduced availability of these minerals and the greater solubility of other minerals. In cattle, Berrett et al. 
Table 6. Pearson correlation coefficients between content and ruminal release of total ash, and individual minerals of different concentrate and forage feedstuffs $(n=24)^{*}$

\begin{tabular}{|c|c|c|c|c|c|c|c|c|c|c|c|c|c|c|}
\hline Item & $\mathrm{Ash}^{+}$ & $\mathrm{CP}^{\neq}$ & $\mathrm{NDF}^{\S}$ & $\mathrm{Ca}$ & $P$ & $\mathrm{Na}$ & K & Mg & $S$ & $\mathrm{Cu}$ & $\mathrm{Zn}$ & $\mathrm{Mn}$ & Co & $\mathrm{Fe}$ \\
\hline Ash & $-0 \cdot 51^{*}$ & $0 \cdot 41^{*}$ & $-0 \cdot 39 *$ & & & & & & & & & & & \\
\hline $\mathrm{Ca}$ & $-0 \cdot 27$ & $-0 \cdot 06$ & $-0 \cdot 20$ & $0 \cdot 49^{*}$ & & & & & & & & & & \\
\hline$P$ & $0 \cdot 52^{*}$ & $0 \cdot 26$ & $-0 \cdot 23$ & $0 \cdot 29$ & $0 \cdot 66^{*}$ & & & & & & & & & \\
\hline $\mathrm{Na}$ & $0 \cdot 34^{*}$ & $0 \cdot 11$ & $-0 \cdot 58^{*}$ & $0 \cdot 10$ & $0 \cdot 49 *$ & $0 \cdot 44^{*}$ & & & & & & & & \\
\hline K & $-0 \cdot 22$ & $-0 \cdot 36^{*}$ & $0 \cdot 56^{*}$ & $0 \cdot 36^{*}$ & $0 \cdot 14$ & $-0 \cdot 21$ & $0 \cdot 18$ & & & & & & & \\
\hline $\mathrm{Mg}$ & $0 \cdot 28$ & $0 \cdot 49 *$ & $-0.75^{*}$ & $0 \cdot 53^{*}$ & $-0 \cdot 13$ & $0 \cdot 48^{*}$ & $-0 \cdot 74^{*}$ & $0 \cdot 54^{*}$ & & & & & & \\
\hline S & $-0 \cdot 08$ & $0 \cdot 29$ & $-0 \cdot 54^{*}$ & $-0 \cdot 16$ & $-0 \cdot 30$ & $0 \cdot 07$ & $-0 \cdot 07$ & $0 \cdot 15$ & $0 \cdot 53^{*}$ & & & & & \\
\hline $\mathrm{Cu}$ & $0 \cdot 48^{*}$ & $0 \cdot 35^{*}$ & $-0.57^{*}$ & $0 \cdot 00$ & $0 \cdot 28$ & $0 \cdot 19$ & $-0 \cdot 38^{*}$ & $0 \cdot 43^{*}$ & $0 \cdot 13$ & $0 \cdot 46^{*}$ & & & & \\
\hline $\mathrm{Zn}$ & $0 \cdot 40^{*}$ & $0 \cdot 29$ & $-0 \cdot 55^{*}$ & $-0 \cdot 11$ & $0 \cdot 34^{*}$ & $0 \cdot 22$ & $-0 \cdot 52^{*}$ & $0 \cdot 19$ & $0 \cdot 30$ & $0 \cdot 39 *$ & $0 \cdot 36^{*}$ & & & \\
\hline Mn & $-0 \cdot 40^{*}$ & $-0 \cdot 46^{*}$ & $0 \cdot 34^{*}$ & $0 \cdot 45^{*}$ & $-0 \cdot 58^{*}$ & $0 \cdot 05$ & $0 \cdot 18$ & $-0 \cdot 15$ & $-0 \cdot 12$ & $-0 \cdot 05$ & $0 \cdot 02$ & $0 \cdot 53 *$ & & \\
\hline Co & $0 \cdot 06$ & $0 \cdot 23$ & $-0 \cdot 42^{*}$ & $0 \cdot 37^{*}$ & $-0 \cdot 17$ & $-0 \cdot 10$ & $-0 \cdot 14$ & $0 \cdot 22$ & $0 \cdot 35^{*}$ & $0 \cdot 42^{*}$ & $0 \cdot 48^{*}$ & $-0 \cdot 04$ & $-0 \cdot 09$ & \\
\hline $\mathrm{Fe}$ & $-0 \cdot 09$ & $0 \cdot 47^{*}$ & $-0.69 *$ & $0 \cdot 00$ & $0 \cdot 13$ & $-0 \cdot 15$ & $-0 \cdot 24$ & $0 \cdot 28$ & $0 \cdot 29$ & $0 \cdot 36^{*}$ & $0 \cdot 46^{*}$ & $-0 \cdot 15$ & $0 \cdot 01$ & $-0 \cdot 11$ \\
\hline
\end{tabular}

$* P<0.05$.

+ Total mineral content

₹ Crude protein.

$\S$ Neutral detergent fibre. 
Table 7. Coefficients of DM abomasal digestion and total ash, and individual mineral abomasal release of concentrate and forage feedstuffs

\begin{tabular}{|c|c|c|c|c|c|c|c|c|c|c|c|c|c|}
\hline Feed & DM* & $\mathrm{Ash}^{+}$ & $\mathrm{Ca}$ & $\mathrm{P}$ & $\mathrm{Na}$ & $\mathrm{K}$ & Mg & $\mathrm{s}$ & $\mathrm{Cu}$ & $\mathrm{Zn}$ & Mn & Co & $\mathrm{Fe}$ \\
\hline Average all feeds & $0 \cdot 68$ & $0 \cdot 82$ & $1 \cdot 00$ & $1 \cdot 00$ & $1 \cdot 00$ & $1 \cdot 00$ & $1 \cdot 00$ & 1.00 & 0.74 & 0.93 & 0.92 & 0.75 & 0.79 \\
\hline Average concentrates & $0 \cdot 71$ & $0 \cdot 91$ & $1 \cdot 00$ & $1 \cdot 00$ & $1 \cdot 00$ & $1 \cdot 00$ & $1 \cdot 00$ & $1 \cdot 00$ & 0.88 & 0.97 & 0.88 & 0.68 & 0.79 \\
\hline Ground ear maize & $0 \cdot 61$ & $0 \cdot 90$ & $1 \cdot 00$ & $1 \cdot 00$ & $1 \cdot 00$ & $1 \cdot 00$ & $1 \cdot 00$ & $1 \cdot 00$ & 0.83 & 0.89 & 0.91 & 0.45 & 0.74 \\
\hline Ground maize & $0 \cdot 82$ & $0 \cdot 95$ & $0 \cdot 99$ & $1 \cdot 00$ & $1 \cdot 00$ & $1 \cdot 00$ & $1 \cdot 00$ & 1.00 & 0.72 & 0.98 & $0 \cdot 70$ & 0.65 & $0 \cdot 18$ \\
\hline Ground sorghum & $0 \cdot 71$ & $0 \cdot 86$ & $1 \cdot 00$ & $1 \cdot 00$ & $1 \cdot 00$ & $1 \cdot 00$ & $1 \cdot 00$ & 1.00 & 0.74 & $1 \cdot 00$ & 0.96 & $0 \cdot 84$ & 0.74 \\
\hline Rice bran & $0 \cdot 70$ & $0 \cdot 67$ & $1 \cdot 00$ & $1 \cdot 00$ & $1 \cdot 00$ & $1 \cdot 00$ & $1 \cdot 00$ & $1 \cdot 00$ & 0.97 & $1 \cdot 00$ & $1 \cdot 00$ & 0.98 & 0.99 \\
\hline Soybean Hulls & $0 \cdot 63$ & $0 \cdot 97$ & $1 \cdot 00$ & $0 \cdot 99$ & $1 \cdot 00$ & 1.00 & $1 \cdot 00$ & 1.00 & $0 \cdot 86$ & 0.92 & 0.43 & $0 \cdot 49$ & 0.77 \\
\hline Wheat bran & $0 \cdot 77$ & $0 \cdot 96$ & $1 \cdot 00$ & $1 \cdot 00$ & $1 \cdot 00$ & 1.00 & $1 \cdot 00$ & 1.00 & 0.96 & $1 \cdot 00$ & 0.99 & $0 \cdot 89$ & $0 \cdot 89$ \\
\hline Maize gluten & $0 \cdot 38$ & $0 \cdot 97$ & $1 \cdot 00$ & $1 \cdot 00$ & $1 \cdot 00$ & 1.00 & $1 \cdot 00$ & 1.00 & 0.88 & 0.94 & 0.79 & 0.44 & $0 \cdot 85$ \\
\hline Cottonseed meal & $0 \cdot 68$ & $0 \cdot 97$ & $1 \cdot 00$ & $1 \cdot 00$ & $1 \cdot 00$ & 1.00 & $1 \cdot 00$ & 1.00 & 0.72 & 0.96 & 0.76 & 0.51 & 0.37 \\
\hline Ground bean & 0.91 & $0 \cdot 99$ & $1 \cdot 00$ & $1 \cdot 00$ & $1 \cdot 00$ & 1.00 & $1 \cdot 00$ & 1.00 & 0.96 & 0.99 & 0.99 & 0.85 & 0.98 \\
\hline Peanut meal & $0 \cdot 87$ & $0 \cdot 73$ & $1 \cdot 00$ & $1 \cdot 00$ & $1 \cdot 00$ & 1.00 & $1 \cdot 00$ & 1.00 & 0.96 & 0.99 & 1.00 & 0.79 & 0.99 \\
\hline Soybean meal & $0 \cdot 88$ & $0 \cdot 98$ & $1 \cdot 00$ & $1 \cdot 00$ & $1 \cdot 00$ & 1.00 & $1 \cdot 00$ & 1.00 & 0.96 & 0.98 & $1 \cdot 00$ & 0.78 & 0.99 \\
\hline Sunflower meal & $0 \cdot 61$ & $0 \cdot 97$ & $1 \cdot 00$ & $1 \cdot 00$ & $1 \cdot 00$ & 1.00 & $1 \cdot 00$ & 1.00 & 0.94 & 0.98 & $1 \cdot 00$ & 0.56 & 0.95 \\
\hline Average forages & 0.65 & $0 \cdot 74$ & $1 \cdot 00$ & $1 \cdot 00$ & 0.99 & 1.00 & $1 \cdot 00$ & $1 \cdot 00$ & 0.60 & 0.90 & 0.97 & $0 \cdot 81$ & 0.79 \\
\hline Maize silage & 0.65 & $0 \cdot 64$ & $1 \cdot 00$ & $1 \cdot 00$ & $1 \cdot 00$ & $1 \cdot 00$ & $1 \cdot 00$ & 1.00 & 0.72 & 0.95 & 0.94 & 0.91 & $0 \cdot 89$ \\
\hline Decumbens & 0.67 & $0 \cdot 77$ & $1 \cdot 00$ & $1 \cdot 00$ & 0.99 & 1.00 & 1.00 & 1.00 & 0.58 & 0.93 & 0.97 & 0.82 & 0.71 \\
\hline Elephant grass & 0.66 & $0 \cdot 60$ & $1 \cdot 00$ & $1 \cdot 00$ & $1 \cdot 00$ & $1 \cdot 00$ & $1 \cdot 00$ & 1.00 & 0.61 & 0.94 & 0.99 & 0.93 & 0.92 \\
\hline Humidicola & $0 \cdot 62$ & $0 \cdot 74$ & $1 \cdot 00$ & $1 \cdot 00$ & $1 \cdot 00$ & $1 \cdot 00$ & $1 \cdot 00$ & $1 \cdot 00$ & 0.79 & 0.90 & $1 \cdot 00$ & 0.79 & $0 \cdot 88$ \\
\hline Marandu & $0 \cdot 67$ & $0 \cdot 77$ & $1 \cdot 00$ & $0 \cdot 98$ & 0.98 & $1 \cdot 00$ & $1 \cdot 00$ & 0.99 & $\cdot 38$ & 0 . & 0.89 & 0.89 & $0 \cdot 71$ \\
\hline MG-4 & $0 \cdot 68$ & $0 \cdot 74$ & $1 \cdot 00$ & $1 \cdot 00$ & 0.99 & $1 \cdot 00$ & $1 \cdot 00$ & $1 \cdot 00$ & 0.72 & 0.91 & 0.96 & 0.98 & $0 \cdot 80$ \\
\hline Mombaça & $0 \cdot 56$ & $0 \cdot 72$ & $1 \cdot 00$ & $1 \cdot 00$ & 0.99 & $1 \cdot 00$ & $1 \cdot 00$ & 1.00 & 0.43 & 0.83 & $1 \cdot 00$ & 0.61 & 0.79 \\
\hline Mulato & $0 \cdot 68$ & $0 \cdot 73$ & $1 \cdot 00$ & $1 \cdot 00$ & 0.99 & 1.00 & $1 \cdot 00$ & 1.00 & 0.65 & 0.82 & 0.94 & 0.83 & $0 \cdot 83$ \\
\hline Peanut forage & $0 \cdot 81$ & $0 \cdot 96$ & $1 \cdot 00$ & $1 \cdot 00$ & $1 \cdot 00$ & $1 \cdot 00$ & $1 \cdot 00$ & 1.00 & 0.72 & 0.92 & $1 \cdot 00$ & 0.90 & $0 \cdot 59$ \\
\hline Tanzania & $0 \cdot 60$ & $0 \cdot 67$ & $1 \cdot 00$ & $1 \cdot 00$ & 0.98 & $1 \cdot 00$ & $1 \cdot 00$ & 1.00 & 0.82 & 0.88 & 0.99 & 0.44 & 0.79 \\
\hline Tifton 85 hay & $0 \cdot 51$ & $0 \cdot 72$ & $1 \cdot 00$ & $1 \cdot 00$ & 0.99 & 1.00 & 1.00 & 1.00 & $0 \cdot 12$ & 0.90 & 0.99 & 0.79 & 0.68 \\
\hline Xaraes & $0 \cdot 68$ & $0 \cdot 76$ & $1 \cdot 00$ & $1 \cdot 00$ & 0.99 & 1.00 & $1 \cdot 00$ & 1.00 & 0.63 & 0.95 & 0.99 & 0.79 & $0 \cdot 82$ \\
\hline
\end{tabular}

* Dry matter.

+ Total mineral content.

(2015) reported solubilities ranging between 0.52 and 0.68 for $\mathrm{Cu}$ and 0.75 and 0.87 for $\mathrm{Zn}$. These values are similar to those reported in the current experiment. Regarding Fe solubility, Lestienne et al. (2005) reported that this reduced value could be related to interactions with phenolic compounds in the feed matrix.

In general, high solubility of minerals was observed after simulated abomasal digestion. As already mentioned, solubility is an essential step to mineral absorption. The absorption coefficients recommended by nutritionists are lower than solubility values reported in the current research, except for $\mathrm{Na}$ and $\mathrm{K}$, which are considered highly soluble. A portion of absorption coefficients described in the literature is related to apparent absorption, which includes the endogenous fraction in the reported values. Costa e Silva et al.
(2015) determined the true absorption coefficients for minerals. Among the minerals evaluated, only the absorption coefficient of Co was close to the average Co release under simulated abomasal conditions. The $\mathrm{Mn}$ and Fe absorption coefficients were close to the ruminal release of these minerals. Mineral homoeostasis is controlled mainly by absorption mechanisms (Field 1981) and may help explain these differences. However, current understanding of the mechanisms for mineral absorption in beef cattle are limited (Spears 2003; Han et al. 2012).

In conclusion, mineral content and ruminal release are highly variable among plant species and between each mineral. In general, ruminal mineral release is high, with the greatest values for $\mathrm{K}$, while $\mathrm{Ca}, \mathrm{S}, \mathrm{Cu}$, $\mathrm{Zn}, \mathrm{Mn}$ and Co have the lowest ruminal release, being proportional to the DM digested. 
Concentrated feeds have a greater mineral release rate than forages. Mineral release is affected by NDF content and mineral concentration. Under simulated abomasal conditions, most minerals are released, and consequently available for absorption. Based on this information, mineral content from basal feed ingredients should be considered as highly available and total mineral content may be used in diet formulation, thereby reducing the level of mineral supplement often routinely used and hence reducing costs and environmental excretion of minerals.

The author gratefully acknowledges the National Council of Scientific and Technological Development (CNPq), National Institute of Science and Technology in Animal Science (INCT - Ciência Animal), Coordination of Improvement of Personal Higher Education (CAPES), and Foundation for Research Support of the State of Minas Gerais (FAPEMIG) for providing a research grant for this research.

\section{REFERENCES}

ADAms, R. S. (1975). Variability in mineral and trace element content of dairy cattle feeds. Journal of Dairy Science $\mathbf{5 8}$, 1538-1548.

AFRC (1993). Energy and Protein Requirements of Ruminants. Wallingford, UK: CAB International.

Amtmann, A. \& Rubio, F. (2012). Potassium in plants. In elS: Citable Reviews in the Life Sciences (Ed. A.M. Hetherington). Chichester, UK: John Wiley \& Sons, Ltd. doi: 10.1002/9780470015902.a0023737. Available online from http://onlinelibrary.wiley.com/doi/10.1002/ 9780470015902.a0023737/abstract (accessed 8 May 2017).

AOAC (2012). Official Methods of Analysis, 19th edn. Arlington, VA, USA: Association of Official Analytical Chemists.

ARC (1980). The Nutrient Requirements of Ruminant Livestock. London, UK: CABI.

Berrett, C. J., Wagner, J.J., Neuhold, K. L., Caldera, E., Seluins, K.S. \& Engle, T.E. (2015). Comparison of National Research Council standards and industry dietary trace mineral supplementation strategies for yearling feedlot steers. The Professional Animal Scientist 31, 237-247.

Bonhomme, A. (1990). Rumen ciliates: their metabolism and relationships with bacteria and their hosts. Animal Feed Science and Technology 30, 203-266.

Braselton, W. E., Stuart, K. J., Mullaney, T. P. \& Herdt, T. H. (1997). Biopsy mineral analysis by inductively coupled plasma atomic emission spectroscopy with ultrasonic nebulization. Journal of Veterinary Diagnostic Investigation 9, 395-400.

Bravo, D., Meschy, F., Bogaert, C. \& Sauvant, D. (2000). Ruminal phosphorus availability from several feeds measured by the nylon bag technique. Reproduction Nutrition Development 40, 149-162.

Broadley, M. R., White, P. J., Hammond, J.P., Zelko, I. \& Lux, A. (2007). Zinc in plants. New Phytologist 173, 677-702.

Čerešñáková, Z., Fľak, P., Poláčiková, M. \& Chrenková, M. (2005). In sacco NDF degradability and mineral release from selected forages in the rumen. Czech Journal of Animal Science 50, 320-328.

Čerešñáková, Z., FĽak, P., Poláčiková, M. \& Chrenková, M. (2007). In sacco macromineral release from selected forages. Czech Journal of Animal Science 52, 175-182.

ChlÁDEK, G. \& ZaPLETAL, D. (2007). A free-choice intake of mineral blocks in beef cows during the grazing season and in winter. Livestock Science 106, 41-46.

CORAH, L. (1996). Trace mineral requirements of grazing cattle. Animal Feed Science and Technology 59, 61-70.

Costa, R. M., Ponsano, E. H. G., Souza, V. C. \& Malafaia, P. (2016). Reduction of phosphorus concentration in mineral supplement on fertility rate, maternal ability and costs of beef cows reared in pastures of Urochloa decumbens. Tropical Animal Health and Production 48, 417422.

Costa e Silva, L. F., Engle, T.E., Valadares Filho, S. C., Rotta, P.P., Valadares, R.F.D., Silva, B.C. \& PACHeCO, M. V.C. (2015). Intake, apparent digestibility, and nutrient requirements for growing Nellore heifers and steers fed two levels of calcium and phosphorus. Livestock Science 181, 17-24.

Demarty, M., Morvan, C. \& Thellier, M. (1984). Calcium and the cell wall. Plant, Cell and Environment 7, 441-448.

Emanuele, S. M. \& Staples, C. R. (1990). Ruminal release of minerals from six forage species. Journal of Animal Science 68, 2052-2060.

Emanuele, S. M., Staples, C. R. \& Wilcox, C. J. (1991). Extent and site of mineral release from six forage species incubated in mobile Dacron bags. Journal of Animal Science 69, 801-810.

Esser, N. M., Hoffman, P. C., Coblentz, W. K., Orth, M. W. \& Weigel, K. A. (2009). The effect of dietary phosphorus on bone development in dairy heifers. Journal of Dairy Science 92, 1741-1749.

FIELD, A. C. (1981). Some thoughts on dietary requirements of macro-elements for ruminants. Proceedings of the Nutrition Society 40, 267-272.

FlaCHOWSKY, G. \& GRÜN, M. (1992). Influence of type of diet and incubation time on major elements release in sacco from Italian ryegrass, untreated and ammonia-treated wheat straw. Animal Feed Science and Technology 36, 239-254.

Flachowsky, G., Grün, M., Polzin, S. \& Kronemann, H. (1994). In sacco dry matter degradability and Ca, Mg and $P$ disappearance from Italian ryegrass, alfalfa hay and wheat straw in sheep and goats. Journal of Animal Physiology and Animal Nutrition 71, 57-64.

Genther, O. N. \& Hansen, S. L. (2014). Effect of dietary trace mineral supplementation and a multi-element trace mineral injection on shipping response and growth performance of beef cattle. Journal of Animal Science 92, 2522-2530 
Han, H., So, H., Domby, E. \& Engle, T. E. (2012). The relationship of pulmonary artery copper concentrations and genes involved in copper homeostasis in cattle, swine, and goats. Asian-Australasian Journal of Animal Sciences 25, 194-199.

He, H., Veneklaas, E. J., Kuo, J. \& Lambers, H. (2014). Physiological and ecological significance of biomineralization in plants. Trends in Plant Science 19, 166-174.

Humer, E. \& Zebeli, Q. (2015). Phytate in feed ingredients and potentials for improving the utilization of phosphorus in ruminant nutrition. Animal Feed Science and Technology 209, 1-15.

Ibrahim, M. N. M., Zemmelink, G. \& Tamminga, S. (1998). Release of mineral elements from tropical feeds during degradation in the rumen. Asian-Australasian Journal of Animal Sciences 11, 530-537.

Jasaitis, D. K., Wohlt, J. E. \& Evans, J. L. (1987). Influence of feed ion content on buffering capacity of ruminant feeds in vitro. Journal of Dairy Science 70, 1391-1403.

Johnson, R.A. \& WICHERN, D.W. (1998). Applied Multivariate Statistical Analysis. New Jersey, NY, USA: Prentice-Hall International.

JunG, H. G. \& Allen, M.S. (1995). Characteristics of plant cell walls affecting intake and digestibility of forages by ruminants. Journal of Animal Science 73, 2774-2790.

Khattree, R. \& NAIK, D. N. (2000). Multivariate Data Reduction and Discrimination. Cary, NC, USA: SAS Institute Inc.

Khorasani, G. R., Janzen, R. A., McGill, W. B. \& Kennelly, J. J. (1997). Site and extent of mineral absorption in lactating cows fed whole-crop cereal grain silage of alfalfa silage. Journal of Animal Science 75, 239-248.

Lestienne, I., Besançon, P. Caporiccio, B., Lullien-Péllerin, V. \& TRÉCHE, S. (2005). Iron and zinc in vitro availability in pearl millet flours (Pennisetum glaucum) with varying phytate, tannin, and fiber contents. Journal of Agricultural and Food Chemistry 53, 3240-3247.

MA, J. F. \& YAmall, N. (2006). Silicon uptake and accumulation in higher plants. Trends in Plant Science 11, 392-397.

MaAthuis, F. J. \& Diatloff, E. (2012). Roles and functions of plant mineral nutrients. In Plant Mineral Nutrients: Methods and Protocols (Ed. F. J. M. Maathuis), pp. 1-21. Methods in Molecular Biology 953. Dordrecht, The Netherlands: Springer.

McBurney, M.I., Allen, M.S. \& Van Soest, P.J. (1986). Praseodymium and copper cation-exchange capacities of neutral-detergent fibres relative to composition and fermentation kinetics. Journal of the Science of Food and Agriculture 37, 666-672.

MERTENS, D. R. (2002). Gravimetric determination of amylase-treated neutral detergent fibre in feeds with refluxing beakers or crucibles: collaborative study. Journal of AOAC International 85, 1217-1240.

Minson, D. J. (1990). Forage in Ruminant Nutrition. San Diego, CA, USA: Academic Press.

Moreira, L. M., Leonel, F. P., Vieira, R. A. M. \& Pereira, J. C. (2013). A new approach about the digestion of fibers by ruminants. Revista Brasileira de Saúde e Produção Animal 14, 382-395.
NRC (2001). Nutrient Requirements of Dairy Cattle, Revised 7th edn. Washington, DC, USA: The National Academy Press.

NRC (2016). Nutrient Requirements of Beef Cattle, 8th edn. Washington, DC, USA: The National Academy Press.

Olubobokun, J. A., CraiG, W. M. \& Pond, K. R. (1990). Effects of mastication and microbial contamination on ruminal in situ forage disappearance. Journal of Animal Science 68, 3371-3381.

Ørskov, E. R., \& McDonald, I. (1979). The estimation of protein degradability in the rumen from incubation measurements weighted according to rate of passage. The Journal of Agricultural Science, Cambridge 92, 499-503.

Perdomo, J.T., Shirley, R. L. \& Chicco, C. F. (1977). Availability of nutrient minerals in four tropical forages fed freshly chopped to sheep. Journal of Animal Science 45, 1114-1119.

Playne, M. J., Echevarría, M. G. \& Megarrity, R. G. (1978). Release of nitrogen, sulphur, phosphorus, calcium, magnesium, potassium and sodium from four tropical hays during their digestion in nylon bags in the rumen. Journal of the Science of Food and Agriculture 29, 520-526.

Pogge, D. J., Drewnoski, M. E. \& Hansen, S. L. (2014). High dietary sulfur decreases the retention of copper, manganese, and zinc in steers. Journal of Animal Science 92, 2182-2191.

Prados, L. F., Valadares Filho, S. C., Santos, S. A., Zanetti, D., Nunes, A. N., Costa, D. R., Mariz, L. D. S., Detmann, E., Amaral, P. M., Rodrigues, F.C. \& Valadares, R. F. D. (2016). Reducing calcium and phosphorus in crossbred beef cattle diets: impacts on productive performance during the growing and finishing phase. Animal Production Science 56, 1643-1649.

Ray, P.P., Jarrett, J. \& Knowlton, K. F. (2013). Effect of dietary phytate on phosphorus digestibility in dairy cows. Journal of Dairy Science 96, 1156-1163.

Sath, K., Pauly, T. \& Holtenius, K. (2012). Mineral balance of Cambodian cattle based on their faecal and urinary excretion. Journal of Animal and Veterinary Advances 11, 4221-4225.

Smart, M. E., Gudmundson, J. \& Christensen, D. A. (1981). Trace mineral deficiencies in cattle: a review. Canadian Veterinary Journal 22, 372-376.

Smith, G.S., Nelson, A. B. \& Boggino, E. J. (1971). Digestibility of forages in vitro as affected by content of 'silica'. Journal of Animal Science 33, 466-471.

Spanghero, M., Zanfi, C., Signor, M., Davanzo, D., Volpe, V. \& Venerus, S. (2015). Effects of plant vegetative stage and field drying time on chemical composition and in vitro ruminal degradation of forage soybean silage. Animal Feed Science and Technology 200, 102-106.

SPEARS, J. W. (1994). Minerals in forages. In Forage Quality, Evaluation, and Utilization (Ed. G.C. Fahey, Jr.), pp. 281-317. Madison, WI, USA: ASA, CSSA, SSSA.

SPEARS, J.W. (1996). Organic trace minerals in ruminant nutrition. Animal Feed Science and Technology 58, 151-163.

SPEARS, J. W. (2003). Trace mineral bioavailability in ruminants. The Journal of Nutrition 133, 1506S-1509S. 
Valadares Filho, S. C., Marcondes, M. I., Chizzotti, M. L. \& Paulino, P. V. R. (2010). Nutrient Requirements of Zebu Beef Cattle - BR-Corte. 2nd edn. Visconde do Rio Branco, Brazil: Suprema Gráfica e Editora.

Van Eys, J. E. \& ReID, R. L. (1987). Ruminal solubility of nitrogen and minerals from fescue and fescue-red clover herbage. Journal of Animal Science 65, 1101-1112.

Yoshihara, Y., Mizuno, H., Ogura, S., Sasaki, T. \& Sato, S. (2013). Increasing the number of plant species in a pasture improves the mineral balance of grazing beef cattle. Animal Feed Science and Technology 179, 138143.

Zanetti, D., Godol, L.A., Estrada, M.M., Engle, T.E., Silva, B. C., Alhadas, H. M., Chizzotti, M. L., Prados, L. F., Renno, L. N. \& Valadares Filho, S. C. (2017). Estimating mineral requirements of Nellore beef bulls fed with or without inorganic mineral supplementation and the influence on mineral balance. Journal of Animal Science 95, 1696-1706. doi: 10.2527/jas2016. 1190. 